\title{
Design and Preliminary Accuracy Studies of an MRI-Guided Transrectal Prostate Intervention System
}

\author{
Axel Krieger ${ }^{1}$, Csaba Csoma ${ }^{1}$, Iulian I. Iordachita ${ }^{1}$, Peter Guion ${ }^{2}$, \\ Anurag K. Singh ${ }^{2}$, Gabor Fichtinger ${ }^{1}$, and Louis L. Whitcomb ${ }^{1}$ \\ ${ }^{1}$ Department of Mechanical Engineering, Johns Hopkins University, Baltimore* \\ ${ }^{2}$ Radiation Oncology Branch, NCI - NIH-DHHS, Bethesda
}

\begin{abstract}
This paper reports a novel system for magnetic resonance imaging (MRI) guided transrectal prostate interventions, such as needle biopsy, fiducial marker placement, and therapy delivery. The system utilizes a hybrid tracking method, comprised of passive fiducial tracking for initial registration and subsequent incremental motion measurement along the degrees of freedom using fiber-optical encoders and mechanical scales. Targeting accuracy of the system is evaluated in prostate phantom experiments. Achieved targeting accuracy and procedure times were found to compare favorably with existing systems using passive and active tracking methods. Moreover, the portable design of the system using only standard MRI image sequences and minimal custom scanner interfacing allows the system to be easily used on different MRI scanners.
\end{abstract}

\section{Introduction}

Background and Motivation: Prostate cancer is the most common noncutaneous cancer in American men. For 2007, Jemal et al. 1] estimate 218,890 new cases of prostate cancer and 27,050 deaths caused by prostate cancer in the United States. The current standard of care for verifying the existence of prostate cancer is transrectal ultrasound (TRUS) guided biopsy. TRUS provides limited diagnostic accuracy and image resolution. In 2] the authors conclude that TRUS is not accurate for tumor localization and therefore the precise identification and sampling of individual cancerous tumor sites is limited. As a result, the sensitivity of TRUS biopsy is only between $60 \%$ and $85 \%$ 34. Magnetic Resonance Imaging (MRI) with an endorectal coil affords images with higher anatomical resolution and contrast than can be obtained using TRUS 2. Targeted biopsies of suspicious areas identified and guided by MRI could potentially increase the sensitivity of prostate biopsies. Moreover, once a lesion is confirmed as cancerous, MR-guided targeted treatment of the lesion with injections of therapeutic agents, cryoablation, or radio frequency (RF) ablation could be used.

\footnotetext{
* The authors gratefully acknowledge support under grants NIH 1R01EB002963 and NSF EEC-9731748.
}

N. Ayache, S. Ourselin, A. Maeder (Eds.): MICCAI 2007, Part II, LNCS 4792, pp. 5967. 2007.

(C) Springer-Verlag Berlin Heidelberg 2007 
Previous Work in MRI Guided Prostate Interventions: MRI guided transperineal prostate biopsy has been demonstrated inside an open MRI scanner [5] and conventional closed configuration MRI scanner 6]. The transrectal approach is generally well tolerated by patients and is considered the standard approach for biopsies. The alternative, transperineal access, requires a longer needle path which may increase patient discomfort. It also generally requires the patient to be sedated for procedures. Beyersdorff et al. report a MRI guided transrectal needle biopsy system, which employs a passive fiducial marker sleeve coaxial with the biopsy needle [7]. In this system, the needle position is manually adjusted while the passive marker is imaged. This approach requires repeated volume imaging of high resolution that takes considerable time to acquire. An endorectal imaging coil can not be used with this system, which compromises the quality of the MR images. Krieger et. al. report a MRI compatible manipulator for transrectal needle biopsy, using an active tracking method comprised of three micro-tracking coils, rigidly attached to the end-effector of the manipulator providing real-time tracking [8. The manipulator contains an endorectal imaging coil and uses two fixed angle needle channels for biopsies of distal and proximal parts of the prostate. However, Krieger et al. identified three disadvantages of this tracking method [9]: (a) The method requires custom scanner programming and interfacing which limits the portability to different scanners. (b) Each tracking coil occupies one receiving scanner channel, limiting the number of imaging coils that can be used simultaneously. (c) Frequent failures in the micro-coils and electrical circuit significantly degrade the reliability of the tracking method. In contrast to these approaches, we have developed a MR guided transrectal prostate interventional system, which employs (a) novel manipulator mechanics with a steerable needle channel in combination with an endorectal imaging coil, (b) a hybrid tracking method, with the goals of shortened procedure time and significantly simplified deployment of the system on different scanners, while achieving millimeter needle placement accuracy.

\section{System Design}

Manipulator Design: The needle manipulator assists the physician in inserting a needle to a predetermined target. A manual actuated design for the manipulator was chosen over an automated design, since the manual actuation reduces development time and approval time for clinical trials. There is a strong current need for an MRI-guided prostate intervention system as a research validation tool. In particular, MR spectroscopy (MRS) and dynamic contrast enhanced (DCE) MRI are two promising developing MR imaging modalities, whose capabilities in finding cancerous lesions in the prostate can be tested using this intervention system. Moreover, manual actuation for insertion of the needle is preferable to many physicians to obtain visual confirmation of the needle alignment before insertion and haptic feedback during the insertion of the needle. Automated insertion of the needle inside the MRI scanner could potentially allow for real-time visualization of the needle insertion and enable detection of 


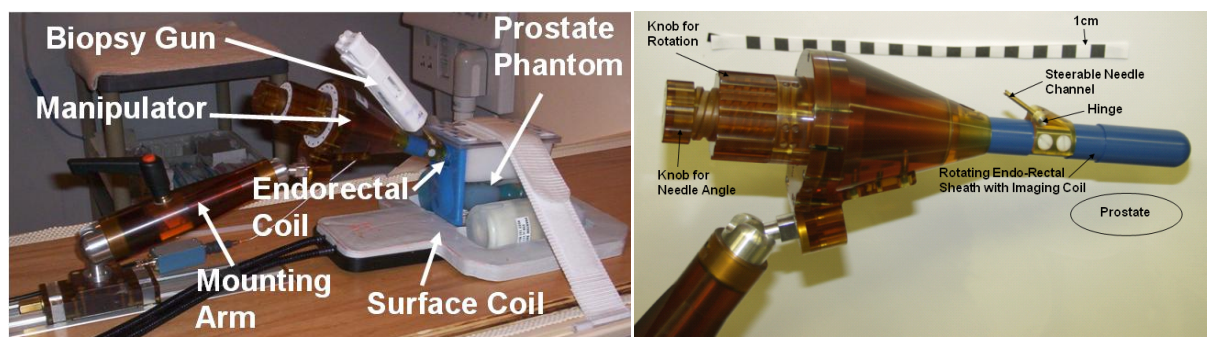

Fig. 1. Left: Photograph of the MRI guided transrectal manipulator with the endorectal imaging coil placed in a prostate phantom. Biopsy gun, surface imaging coil and mounting arm are also visible. Right: Closeup photograph of the manipulator. Turning the knobs on the left rotate the endorectal sheath with hinge and needle channel and change the angle of the steerable needle channel respectively. An endorectal, single loop imaging coil is integrated into the sheath.

prostate deformation, misalignment, and deflection of the needle. However, the design for a fully automated manipulator for prostate biopsy would be further complicated by the fact that the tissue specimen has to be removed after each biopsy, which is hard to achieve automatically.

In our design, the patient is pulled out of the MRI scanner on the scanner table for the physician to manually actuate the manipulator and insert the biopsy needle. Figure 1 on the left shows the manipulator with its endorectal imaging coil placed in a prostate phantom (CIRS Inc, Norfolk, VA). The position of the manipulator is secured using a mounting arm. The manipulator guides the needle tip of a standard MR compatible biopsy gun (Invivo Germany GmbH, Schwerin, Germany) to a predetermined target in the prostate. A surface imaging coil is placed under the phantom to enhance the MRI signal.

Figure 1 on the right shows a close up photograph of the manipulator. The endorectal sheath is inserted in the rectum, such that the hinge is placed close to the anus of the patient. The endorectal sheath contains a single loop imaging coil, which is glued into a machined groove on the sheath. A steerable needle channel is integrated into the sheath. The three degrees of freedom (DOF) to reach a target in the prostate are rotation of the sheath, angulation change of the steerable needle channel, and insertion of the needle. Rotation of the sheath is achieved by turning the larger diameter knob on the left of the manipulator, which directly rotates the sheath with hinge and needle channel. An internal spring washer applies sufficient axial pre-load to avoid unintentional rotation of the sheath. The sheath can be rotated 360 degrees, thus allowing for a variety of patient positions including prone, supine and decubitus. This is further supported by the cone shape of the manipulator, which precludes obstructions for the biopsy gun at all rotational angles (except for the attachment to the mounting arm). In Figure 11 an outline of a prostate is sketched below the sheath, indicating prone positioning of the patient. Needle angle adjustment of the steerable needle channel is controlled by turning the smaller diameter knob on the left of the manipulator. Turning the knob causes an internal rod in the center of the 
manipulator axis to be translated forward and backward via a two stage cumulative screw mechanism. The push rod is connected to the steerable needle channel, thus rotating the needle channel about the hinge axis and controlling the needle angle. A narrow slot on the bottom of the endorectal sheath allows the needle to exit the sheath at an angle between 17.5 and 40 degrees.

The mounting arm consists of two parts: a slide and rail assembly (Igus Inc., E. Province, RI) for linear motion into and out of the scanner bore with an integrated locking mechanism and a custom designed passive arm. The passive arm is comprised of a rigid plastic rod connected with spherical joints to the slide and the manipulator respectively. A locking mechanism is built into the rod to simultaneously immobilize both joints, once the manipulator is placed at its desired location. The mounting arm is designed to be sufficiently strong and rigid to practically preclude deflection of the manipulator, thus allowing an initial registration of the manipulator position to hold during an interventional procedure. The endorectal sheath with the hinge and needle channel are cleaned and sterilized before every procedure. Medical grade heat shrink (Tyco Electronics Corporation, Menlo Park, CA) is fitted around the sheath to keep it cleaner during a procedure. A click-in mechanism, comprised of a flat spring and a small nylon ball provides fast and easy assembly of the sterilized parts to the manipulator prior to a procedure.

The presence of a strong magnetic field inside an MRI scanner precludes the use of any ferromagnetic materials. Nonmagnetic metals can create imaging artifact, caused by a disturbance of the magnetic field due to difference in susceptibility of the metal and surrounding objects, and need to be minimized. The manipulator is constructed mostly of plastic materials, foremost of Ultem (GE Plastics, Pittsfield, MA), selected for its structural stability, machinability and low cost. The endorectal sheath is built out of medical grade Ultem, since it may contact patient tissue. Only very small nonmagnetic metallic components are placed closed to the field of view (FOV) of the prostate: a brass needle channel, a phosphor bronze flat spring for the click in mechanism of the sheath, and an aluminum hinge axle. Additional brass and aluminum parts located in the mounting arm are spaced sufficiently from the FOV. Imaging studies revealed that the device did not cause visual artifacts at the FOV.

Hybrid Tracking Method: The hybrid tracking method is comprised of a combination of passive tracking and joint encoders - an approach similar to that reported in [9]. At the beginning of an interventional procedure, the initial position of the device in scanner coordinates is obtained by automatically segmenting fiducial markers placed on the device in MRI images. From this initial position motion of the device along its DOFs is encoded with fiber-optical and manual encoders. For initial registration, an attachment is placed concentrically over the needle channel of the manipulator (Figure 2 left). The attachment contains two tubular MRI markers (Beekley Corp., Bristol, CT). Two additional markers are placed into the main axis of the manipulator. Instead of acquiring axial image sets along the axes, which would take several minutes, a thin slab of $1 \mathrm{~mm} x$ $1 \mathrm{~mm}$ x $1 \mathrm{~mm}$ isotropic sagittal turbo spin echo (TSE) proton density images 

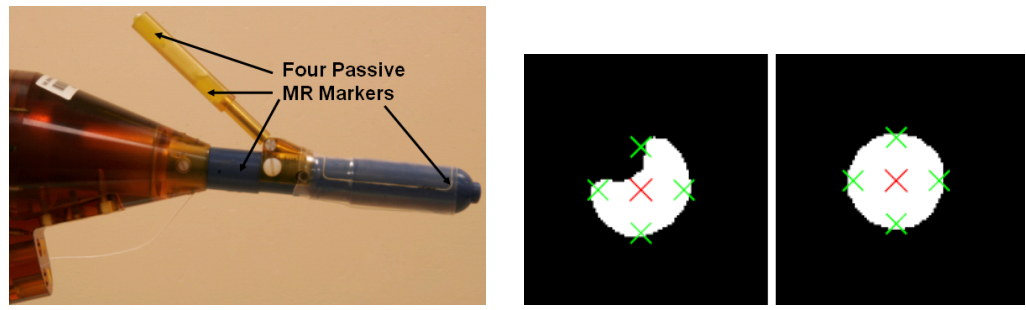

Fig. 2. Left: Photograph of manipulator during initial registration. An attachment is placed concentrically over the needle channel. The tube contains two tubular markers. Two additional markers are placed into the main axis of the manipulator. Right: Example of two binary reformatted MR images axial to a fiducial marker. The segmentation algorithm finds the best fitting circle center indicated by a big cross on both images. The algorithm is able to find the center, even when air bubbles in the marker on the left contaminate the image. Small crosses indicate the border of the marker.

in the plane of the markers is obtained. This reduces the imaged volume significantly and therefore reduces scan time of this high resolution image set to 2.5 minutes. In order to aid automatic segmentation of the markers, the sagittal images are reformatted using a custom targeting program as axial images along the main axis of the device and along the needle axis. The tubular markers appear on the reformatted axial images as circles. An algorithm was written based on the Hough transformation, which finds on each binary reformatted image the best fitting center of a circle with known diameter of the marker (Figure 2, right). This segmentation is very robust even on images containing air bubbles in the marker. Once both axes are calculated from the circle centers using a least square minimization, the 6-DOF position of the manipulator is defined.

Rotation and needle angle change are redundantly encoded by MRI-compatible fiber-optic encoders and mechanical scales placed on the actuation knobs of the manipulator. The needle insertion depth is read manually using the scale on the needle. Although not present in our current design, it is possible to incorporate a translational optical encoder for the needle insertion. The fiber optic joint encoders consist of photoelectric sensors (Banner Engineering Corp., Minneapolis, Minnesota) placed in a box in the control room, adjacent to the shielded MRI scanner room. Each sensor is connected to two plastic optical fibers: one for sending of optical signal, and one for reception of optical signal. Only the plastic optical fibers are passed into the scanner room. Full MR compatibility of the joint encoding is achieved, since no electrical signal or power cables are passed into the scanner room. The optical fiber ends of each sensor are placed opposing each other through a code wheel for encoding rotation of the manipulator and through a code strip for encoding translation of the push rod, and thus indirectly encoding needle angle change. A two channel quadrature design with a third channel as index pulse is used for both encoders. Each sensor provides one channel, so six sensors are necessary to build the two encoders. Encoder 
resolution for rotation of the manipulator is 0.25 degrees, and for needle angle less than 0.1 degrees at all needle angles. In our present design the resolution of the encoders is limited by the size of the core diameter $(0.25 \mathrm{~mm})$ of the plastic fiber, since the light is not columnated before passing through the code wheel.

Targeting Program: The targeting program runs on a laptop computer located in the control room. The only data transfer between laptop and scanner computer are DICOM image transfers. The fiber optic encoders interface via a USB counter (USDigital, Vancouver, Washington) to the laptop computer. The targeting software displays the acquired MR images, provides the automatic segmentation for the initial registration of the manipulator, allows the physician to select targets for needle placements, provides targeting parameters for the placement of the needle, and tracks rotation and needle angle change provided by the encoders, while the manipulator is moved on target.

\section{Experiments, Results, and Discussion}

The system for MRI guided transrectal prostate interventions was tested in a phantom experiment on a 3T Philips Intera MRI scanner (Philips Medical Systems, Best, NL) using standard MR compatible biopsy needles and non artifact producing glass needles. The experimental setup is shown in Figure 1

Biopsy Needle Accuracies: The manipulator was placed in a prostate phantom and its initial position was registered. Twelve targets were selected within all areas of the prostate, from base to mid gland to apex, on T2 weighted axial TSE images (Figure 3. first row). For each target, the targeting program calculated the necessary targeting parameters for the needle placement. Rotation, needle angle and insertion depth to reach the target were displayed on a window which was projected onto a screen located next to the scanner. The phantom was pulled out of the MRI scanner on the scanner table, the physician rotated the manipulator, adjusted the needle angle and inserted the biopsy needle according to the displayed parameters. Since the fiber optic cables of our present prototype were too short to reach all the way from the control room into the scanner, this experiment was performed without the use of optical encoders. Instead, solely the mechanical scales on the manipulator were used to encode the rotation and needle angle. Compared to the respective resolution of the optical encoders of 0.25 degrees and 0.1 degrees, the mechanical scales feature slightly lower resolutions of 1.0 degrees and 0.5 degrees. The phantom was rolled back into the scanner to confirm the location of the needle on axial TSE proton density images which show the void created by the biopsy needle tip close to the target point (Figure 3, second row). The in-plane error for each of the twelve biopsies, defined as the distance of the target to the biopsy needle line was subsequently calculated to assess the accuracy of the system. The needle line was defined by finding the first and the last slice of the acquired confirmation volume, where the needle void is clearly visible. The center of the needle void on the first slice and the center of the void on the last slice define the needle line. The out of 


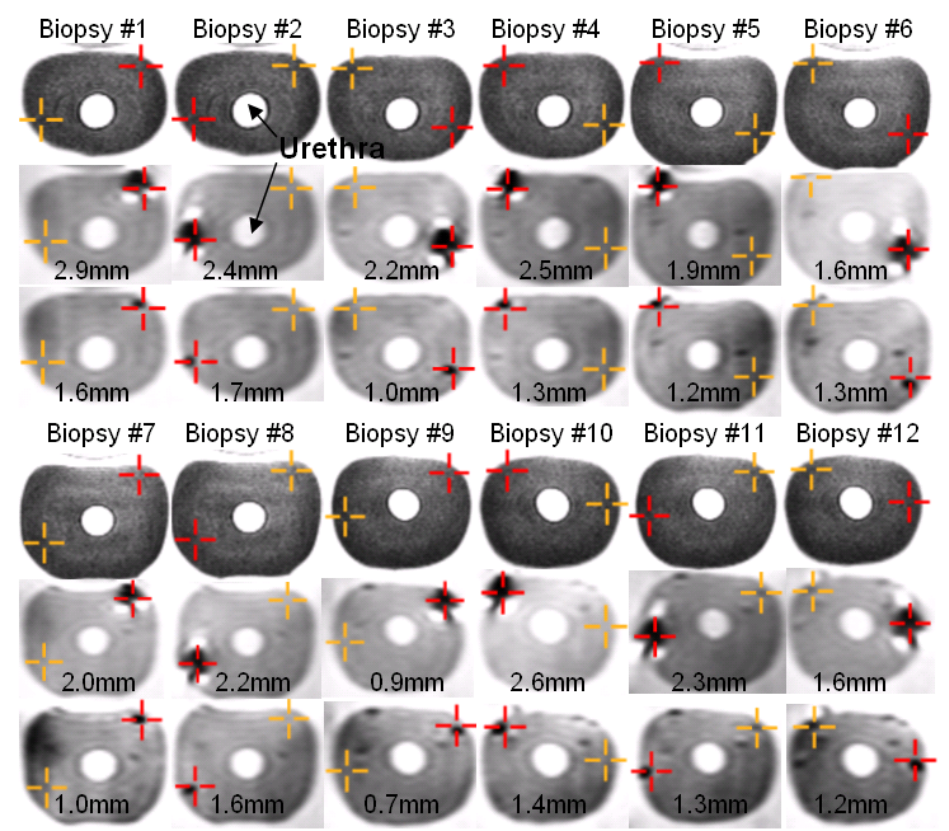

Fig. 3. Targeting images, biopsy needle confirmation images, glass needle confirmation images and in plane errors for twelve biopsies of a prostate phantom. First and fourth row: Two targets (cross hairs) per image are selected on axial TSE T2-weighted images. The dark cross hair represents the active target. Second and fifth row: The biopsy needle tip void is visualized in an axial TSE proton density image. The desired target approximately matches the actual position of the needle. Third and sixth row: The glass needle tip void is visualized in an axial TSE proton density image. The void for the glass needle is much smaller than for the biopsy needle and closer to the selected target. Numbers indicate the in-plane needle targeting error for the needle placement.

plane error is not critical in biopsy procedures due to the length of the biopsy core and was not calculated. Hence, from the purpose of accuracy, there is no need for a more precise motorized needle insertion. The average in-plane error for the biopsy needles was $2.1 \mathrm{~mm}$ with a maximum error of $2.9 \mathrm{~mm}$.

Glass Needle Accuracies: The void created by the biopsy needle is mostly due to susceptibility artifact caused by the metallic needle. The void is not concentric around the biopsy needle and depends on the orientation of the needle to the direction of the main magnetic field in the scanner (B0), and the direction of the spatially encoding magnetic field gradients $[10$. Consequently, center of needle voids do not necessarily correspond to actual needle centers. And since the same imaging sequence and similar orientation of the needle is used for all targets in a procedure, a systematic shift between needle void and actual needle might occur, which introduces a bias in the accuracy calculations. To explore this theory, every biopsy needle placement in the prostate phantom was followed by a placement of a glass needle to the same depth. The void created by the glass needle is 
purely caused by a lack of protons in the glass compared to the surrounding tissue, and is thus artifact free and concentric to the needle. The location of the glass needle was again confirmed by acquiring axial TSE proton density images (Figure 3, third row). The average in-plane error for the glass needles was 1.3 $\mathrm{mm}$ with a maximum error of $1.7 \mathrm{~mm}$, compared to $2.1 \mathrm{~mm}$ and $2.9 \mathrm{~mm}$ for the biopsy needles, which is sufficient to target the minimal clinically significant foci size of $1 / 2$ cc 11 . Analyzing the error reveals an average shift between glass needle void location and biopsy needle void location of only $0.1 \mathrm{~mm}$ in the L-R direction, but $0.9 \mathrm{~mm}$ in the $\mathrm{A}-\mathrm{P}$ direction. This corresponds to the direction of the frequency encoding gradient of the TSE imaging sequence and is consistent with the findings of [10]. The procedure time for six needle biopsies not including the glass needle insertion was measured at 45 minutes.

In summary, we reported the results of preliminary phantom experiments to evaluate the feasibility of performing prostate interventions with the proposed system. The phantom experiments show adequate coverage of the prostate gland and demonstrate accurate and fast needle targeting of the complete clinical target volume. The errors and procedure time compare favorably to reported results (average error $1.8 \mathrm{~mm}$ and average procedure times of 76 minutes) that Krieger et al. achieved with the active tracking method in initial clinical trials [4]. The hybrid tracking method allows this system to be used on any MRI scanner without extensive systems integration and calibration. The two connections required are connection of the endorectal imaging coil to a scanner receiver channel and the DICOM image transfer between scanner computer and laptop computer running the targeting program. The rigid construction of mounting arm and manipulator, optimized manipulator mechanics, and use of fast actuated biopsy guns suggest that reported phantom accuracies of the proposed system translate well to real anatomical accuracies in clinical studies. Institutional review board (IRB) approvals were granted at two clinical sites. Initial clinical results will be reported at the conference.

\section{References}

1. Jemal, A., Siegel, R., Ward, E., Murray, T., Xu, J., Thun, M.J.: Cancer statistics, 2007. CA Cancer J. Clin. 57(1), 43-66 (2007)

2. Yu, K.K., Hricak, H.: Imaging prostate cancer. Radiol. Clin. North. Am. 38(1), 59-85 (2000)

3. Norberg, M., Egevad, L., Holmberg, L., Sparén, P., Norlén, B.J., Busch, C.: The sextant protocol for ultrasound-guided core biopsies of the prostate underestimates the presence of cancer. Urology 50(4), 562-566 (1997)

4. Terris, M.K.: Sensitivity and specificity of sextant biopsies in the detection of prostate cancer: preliminary report. Urology 54(3), 486-489 (1999)

5. Hata, N., Jinzaki, M., Kacher, D., Cormak, R., Gering, D., Nabavi, A., Silverman, S.G., D'Amico, A.V., Kikinis, R., Jolesz, F.A., Tempany, C.M.: Mr imaging-guided prostate biopsy with surgical navigation software: device validation and feasibility. Radiology 220(1), 263-268 (2001) 
6. Susil, R.C., Camphausen, K., Choyke, P., McVeigh, E.R., Gustafson, G.S., Ning, H., Miller, R.W., Atalar, E., Coleman, C.N., Ménard, C.: System for prostate brachytherapy and biopsy in a standard $1.5 \mathrm{t}$ mri scanner. Magn. Reson. Med. 52(3), 683-687 (2004)

7. Beyersdorff, D., Winkel, A., Hamm, B., Lenk, S., Loening, S.A., Taupitz, M.: Mr imaging-guided prostate biopsy with a closed $\mathrm{mr}$ unit at $1.5 \mathrm{t}$ : initial results. Radiology 234(2), 576-581 (2005)

8. Krieger, A., Susil, R.C., Menard, C., Coleman, J.A., Fichtinger, G., Atalar, E., Whitcomb, L.L.: Design of a novel MRI compatible manipulator for image guided prostate interventions. IEEE Transactions on Biomedical Engineering 52(2), 306$313(2005)$

9. Krieger, A., Metzger, G., Fichtinger, G., Atalar, E., Whitcomb, L.L.: A hybrid method for 6-DOF tracking of MRI-compatible robotic interventional devices. In: Proceedings - IEEE International Conference on Robotics and Automation, Orlando, FL, United States, vol. 2006, pp. 3844-3849. IEEE Computer Society Press, Los Alamitos (2006)

10. DiMaio, S.P., Kacher, D.F., Ellis, R.E., Fichtinger, G., Hata, N., Zientara, G.P., Panych, L.P., Kikinis, R., Jolesz, F.A.: Needle artifact localization in 3t mr images. Stud. Health. Technol. Inform. 119, 120-125 (2006)

11. Bak, J.B., Landas, S.K., Haas, G.P.: Characterization of prostate cancer missed by sextant biopsy. Clin. Prostate. Cancer 2(2), 115-118 (2003) 